\title{
Comparison of efficacy and safety between pembrolizumab combined with chemotherapy and simple chemotherapy in neoadjuvant therapy for esophageal squamous cell carcinoma
}

\author{
Bingjiang Huang ${ }^{1}$, Haiyan Shi ${ }^{2}$, Xiaohua Gong ${ }^{2}$, Jing Yu$^{3}$, Caixia Xiao ${ }^{3}$, Bin Zhou ${ }^{3}$, Zibin Liang ${ }^{1 "}$, \\ Xiaojian Li ${ }^{1 \#}$
}

${ }^{1}$ Department of Thoracic Oncology, The Cancer Center of the Fifth Affiliated Hospital of Sun Yat-sen University, Zhuhai, China; ${ }^{2}$ Department of Cardiothoracic Surgery, The Fifth Affiliated Hospital of Sun Yat-sen University, Zhuhai, China; ${ }^{3}$ Department of Minimally Invasive Surgery, The Cancer Center of The Fifth Affiliated Hospital of Sun Yat-sen University, Zhuhai, China

Contributions: (I) Conception and design: B Huang, Z Liang; (II) Administrative support: X Li, X Gong; (III) Provision of study materials or patients: B Huang, X Li; (IV) Collection and assembly of data: H Shi, J Yu, C Xiao, B Zhou; (V) Data analysis and interpretation: B Huang; (VI) Manuscript writing: All authors; (VII) Final approval of manuscript: All authors.

\#These authors contributed equally to this work.

Correspondence to: Zibin Liang; Xiaojian Li. Department of Thoracic Oncology, The Cancer Center of the Fifth Affiliated Hospital of Sun Yat-sen University, Zhuhai 519020, China. Email: liangzb@mail.sysu.edu.cn; 7008351@qq.com.

Background: Immunotherapy can activate the recognition of tumor antigen, build immune memory, and more and more clinical trials have taken the scheme of immunochemotherapy or immunoradiotherapy as a treatment strategy for esophageal squamous cell carcinoma (ESCC). Our objective was to compare the efficacy and safety between pembrolizumab combined with the chemotherapy group and simple chemotherapy in neoadjuvant therapy of ESCC.

Methods: Fifty-four ESCC patients with stage II-IVa were enrolled at the Fifth Affiliated Hospital of Sun Yat-sen University between January 2018 and December 2020, including 23 in the pembrolizumab combined with chemotherapy group (combined group), and 31 in the simple chemotherapy group. All patients received radical surgical treatment after two cycles of neoadjuvant therapy.

Results: The pathological complete response (pCR) and objective response rate (ORR) in the combined group were significantly higher than that of the simple chemotherapy group $(30.4 \%$ vs. 9.7\%, $\mathrm{P}=0.048$; $86.9 \%$ vs. $95.7 \%, \mathrm{P}=0.017)$ as well as the score of tumor regression $\geq 2(80.7 \%$ vs. $50.0 \%, \mathrm{P}=0.013)$. And the complete rate of esophagectomy and R0 /R1 resection rate in the two groups were not statistically significant. Otherwise, the incidence of adverse events in the combined group was similar compared with the simple chemotherapy group.

Conclusions: Pembrolizumab combined with chemotherapy showed promising activity with a manageable safety profile. And it could offer a potential new neoadjuvant treatment approach for patients with ESCC.

Keywords: Esophageal squamous cell carcinoma (ESCC); neoadjuvant therapy; pembrolizumab; docetaxel; nidaplatin

Submitted Sep 07, 2021. Accepted for publication Oct 19, 2021.

doi: 10.21037/jgo-21-610

View this article at: https://dx.doi.org/10.21037/jgo-21-610 


\section{Introduction}

Esophageal cancer is increasing year by year in China. By 2020, the incidence rate of esophageal cancer was sixth, with a mortality rate of fourth, and it was eighth and sixth worldwide (1). Furthermore, the proportion of esophageal squamous cell carcinoma (ESCC) in China was more than 90\%. ESCC risk factors includes smoking, alcohol, hot drinks, and nitrosamine intake. In addition, micronutrient also related to the occurrence of ESCC, such as vitamin $\mathrm{C}$, vitamin $\mathrm{E}$ and folic acid. Also genetic has very big effect on ESCC (2). Many clinical studies have shown that the prognosis of ESCC after neoadjuvant chemotherapy is better than simple radical surgical treatment The Japanese JCOG9204 and JCOG9907 trials have prompted a transition from postoperative adjuvant therapy to neoadjuvant therapy in cII/cIII ESCC patients (3). However, nearly half of the patients who have underwent neoadjuvant chemotherapy and surgery will relapse within 2 years, become the bottleneck of the treatment in ESCC, also the overall survival rate in 5 years is still not optimistic (4-7). Professor Liu's NEOCRTEC5010 study established the position of neoadjuvant chemoradiotherapy in locally advanced ESCC in China (8). Trials showed that neoadjuvant chemoradiotherapy improved the OS compared with all other treatment modalities, including neoadjuvant chemotherapy, neoadjuvant radiotherapy and surgery alone. However, the risk of postoperative mortality after neoadjuvant chemoradiotherapy also increased significantly compared with that of surgery alone or neoadjuvant chemotherapy + surgery (9). So patients who received neoadjuvant chemoradiotherapy are relatively less and are more likely to receive neoadjuvant chemotherapy. Otherwise, immunotherapy can activate the recognition of tumor antigen, build immune memory, and play a role after surgical resection of the tumor. And it demonstrated that experimentally infected ESCC responds by increasing the expression of $\mathrm{B} 7-\mathrm{H} 4$ and lysine demethylase $5 \mathrm{~B}$, which allowed subsequent in vivo analysis of the immunotherapeutic effects of antiB7-H4 and histone demethylase inhibitors in models of chronic infection and immunity against xenografted human tumors (10). Pembrolizumab combined with platinum-based chemotherapy has been approved for the first-line treatment of unresectable or unsuitable for radical chemotherapy for locally advanced or metastatic esophageal cancer or gastroesophageal junction cancer. Nowadays, immunotherapy is added to neoadjuvant therapy in clinical trials, taking the neoadjuvant scheme of immunochemotherapy or immunoradiotherapy as a treatment strategy for ESCC. But till now the reports of efficacy and safety are poor, and there is no evidence for obvious benefit of neoadjuvant immunotherapy for ESCC. With progress in basic and clinical research on immunotherapy, optimal preoperative neoadjuvant therapy for locally advanced ESCC will likely be established in the future. So it is necessary to further study the efficacy and safety of neoadjuvant immunochemotherapy for ESCC $(8,11)$. Otherwise, docetaxel combined with nedaplatin has the advantage of higher clinical efficacy and lower adverse effects in the treatment of ESCC. This retrospective study aimed to compare the efficacy and safety of pembrolizumab combined chemotherapy and simple chemotherapy in neoadjuvant treatment of locally advanced ESCC.

We present the following article in accordance with the STROBE reporting checklist (available at https://dx.doi. org/10.21037/jgo-21-610).

\section{Methods}

\section{Patient characteristics}

This study included patients with stage II-IVa ESCC admitted to the Fifth Affiliated Hospital of Sun Yat-sen University from January 2018 to December 2020. The criteria included: (I) diagnosed by endoscopic pathology; (II) unresectable or difficult to completely resect; (III) PS score was 1-2 points. The exclusion criteria were: (I) age over 75 years; (II) with severe cardiac and pulmonary dysfunction or various diseases that were not tolerable to intravenous chemotherapy; (III) there were immune diseases or were unsuitable for immunotherapy in the active period of hepatitis B; (IV) cervical esophageal cancer, other malignant tumors or multiple sources of malignant tumors were diagnosed within 5 years; (V) without chemotherapy, surgery or traditional Chinese medicine treatment before treatment; (VI) related clinical data was incomplete. Sixtyone patients who met the inclusion criteria were randomly divided into the combination group (23 cases) and the simple chemotherapy group (31 cases).

\section{Patient treatment}

Neoadjuvant immunotherapy combined chemotherapy (combined group): (I) docetaxel $75 \mathrm{mg} / \mathrm{m}^{2}+$ nidaplatin $80 \mathrm{mg} / \mathrm{m}^{2}$, intravenous drip, d1; (II) pembrolizumab $200 \mathrm{mg}$, 
intravenous infusion, $\mathrm{d} 2$.

Neoadjuvant chemotherapy (simple chemotherapy group): (I) docetaxel $75 \mathrm{mg} / \mathrm{m}^{2}+$ nidaplatin $80 \mathrm{mg} / \mathrm{m}^{2}$, intravenous drip, $\mathrm{d} 1$.

All patients were treated for 21 days as a cycle, and surgery was performed 4-6 weeks after two cycles. Before the operation, the whole-body imaging examination and evaluation were performed before the operation, including neck, chest, upper abdomen contrast-enhanced CT, and upper gastrointestinal angiography.

All patients underwent single port pneumatic mediastinoscopic synchronous laparoscopic radical esophagectomy. Before the operation, indocyanine green was injected under a gastroscope to trace mediastinal lymph nodes. There was no residual intraoperative carcinoma in all operations. Postoperative pathological examination was needed to determine whether the cutting edge was negative.

According to RECIST 1.1 (12), the outcome was divided into complete response (CR), partial response (PR), stable disease (SD), and progressive disease (PD). The proportion of $\mathrm{CR}+\mathrm{PR}$ was objective response rate (ORR), and the proportion of $\mathrm{CR}+\mathrm{PR}+\mathrm{SD}$ was disease control rate (DCR). Adverse events were any adverse clinical events that occurred in treatment, and were evaluated according to CTCAE 4.0.

The tumor regression degree was evaluated by the proportion of scar and residual tumor, and it was grading into 5 degrees according to Mandard's TRG system (13): grade 0 is no regression, grade 1 is residual tumor $>50 \%$, grade 2 is residual tumor $10-50 \%$, grade 3 is residual tumor $<10 \%$, grade 4 is no residual tumor.

\section{Statistical analysis}

SPSS 22.0 software was used for statistical analysis. The continuous variables per the normal distribution were expressed by means \pm standard deviation, and a $t$-test was used. The classified variables were expressed by cases (\%), and the chi-square test or Fisher exact probability method was used. The ordered variables and the continuous variables not in accordance with the normal distribution were tested by rank-sum test. $\mathrm{P}<0.05$ were considered statistically significant.

\section{Ethical statement}

All procedures performed in this study involving human participants were in accordance with the Declaration of
Helsinki (as revised in 2013). The study was approved by ethics board of Fifth Affiliated Hospital of Sun Yat-sen University (No. K248-1) and informed consent was taken from all the patients.

\section{Results}

As shown in Table 1, there was no significant difference in gender, age, tumor location, the stage before chemotherapy, tumor length, squamous cell carcinoma differentiation, and PD-L1 expression between the two groups.

After two-cycle neoadjuvant therapy, CR was observed in 8 cases ( 7 cases of postoperative pathology were pCR), 13 cases of PR, 2 cases of SD, 1 case of PD, and ORR, DcR was $86.9 \%$ (20/23), $95.7 \%$ (22/23) respectively in the combined group. However, in the chemotherapy group, CR was observed in 3 cases ( 3 cases of postoperative pathology were pCR), 14 cases of PR, 10 cases of SD, 4 cases of PD, and ORR, DcR was 54.9\% (17/31), 87.1\% (27/31). pCR $(\mathrm{P}=0.048)$ and $\mathrm{ORR}(\mathrm{P}=0.017)$ were significantly different between the two groups (Table 2).

Surgery was performed 4-6 weeks after two cycles of neoadjuvant therapy. There were 2 patients in the combination group and 4 patients in the chemotherapy group (6.7\% and $12.9 \%$ respectively) who did not undergo surgery due to the progression of lesions or serious complications. Forty-seven patients underwent esophagectomy. The R0 (no residual tumor at the cutting edge under the naked eye and microscope; R1 means residual tumor at the cutting edge under the microscope. The R0 resection rate of the combination group was $100 \%(21 / 21)$, and that of the chemotherapy group was $96.3 \%(26 / 27)$. In the combined group, 1 case of the tracheoesophageal fistula was not resected, and 1 case of SD patient with thoracoscopic surgery found that the tumor invaded the trachea, so the operation was stopped. In the chemotherapy group, 1 patient vomited blood, 1 refused follow-up treatment after grade 4 marrow suppression, and 1 refused surgery and underwent radical radiotherapy. The complete rate of esophagectomy and R0 /R1 resection rate in the two groups were not statistically significant (Table 3).

And it was observed that in the combined group, there were 3 cases of grade 0, 2 cases of grade 1, 5 cases of grade 2, 5 cases of grade 3 , and 6 cases of grade 4 . While, in the chemotherapy group, there were 6 cases of grade 0,7 cases of grade 1, 5 cases of grade 2, 5 cases of grade 3, and 3 cases of grade 4 . The score of tumor regression $\geq 2$ was statistically significant between the two groups (Table 4). 
Table 1 Clinical characteristic of squamous cell carcinoma before chemotherapy

\begin{tabular}{|c|c|c|c|}
\hline Characteristics & Combined group (\%) & Chemotherapy group (\%) & $P$ value \\
\hline Male & $21(91.3)$ & $30(96.7)$ & \\
\hline Female & $2(8.7)$ & $1(3.3)$ & \\
\hline Age $(\bar{x} \pm \mathrm{s}$, years $)$ & $59.2 \pm 7.3$ & $58.9 \pm 6.4$ & 0.8649 \\
\hline Upper-thoracic & $4(17.4)$ & $2(6.4)$ & \\
\hline Middle-thoracic & $13(56.5)$ & $22(50.0)$ & \\
\hline Lower-thoracic & $6(26.1)$ & $7(22.6)$ & \\
\hline T stage & & & 0.431 \\
\hline $\mathrm{T} 4$ & $4(17.4)$ & $3(9.7)$ & \\
\hline $\mathrm{N}$ stage & & & 0287 \\
\hline $\mathrm{N} 1$ & $4(17.4)$ & $10(32.3)$ & \\
\hline N2 & $14(60.9)$ & $18(58.1)$ & \\
\hline N3 & $5(21.7)$ & $3(9.6)$ & \\
\hline Clinical staging & & & 0.291 \\
\hline II & $3(13.0)$ & $4(12.9)$ & \\
\hline III & $14(60.8)$ & $22(70.1)$ & \\
\hline G3 & $13(56.5)$ & $22(71.0)$ & \\
\hline Expression of PD-L1 & & & 0.476 \\
\hline Positive & $8(34.7)$ & $6(19.4)$ & \\
\hline Negative & $7(30.4)$ & $13(41.9)$ & \\
\hline Unknown & $8(34.7)$ & $12(38.7)$ & \\
\hline
\end{tabular}

The indication of neoadjuvant therapy for ESCC in our study was clinical-stage T2-4aN1-3 (AJCC 8 TNM classification). PD-L1 $\geq 1 \%$ was considered positive, and $<1 \%$ was considered negative. The classified variables were expressed by cases (\%), and the chi-square test or Fisher exact probability method was used. ESCC, esophageal squamous cell carcinoma.

To evaluate the safety of the two groups, we further observed the adverse effects of the patients. The incidence of hematological toxicity above grade 3 was $13.0 \%(3 / 23)$ and $12.9 \%(4 / 31) \mathrm{P}=0.838$ in the combination group and simple chemotherapy group respectively, and that of nonhematological toxicity above grade 3 was $20.0 \%(6 / 30)$ and $12.9 \%(4 / 31) \mathrm{P}=0.724$ in the combination group. Hypothyroidism (or hyperthyroidism), rash, immune 
Table 2 Comparison of two groups after neoadjuvant therapy

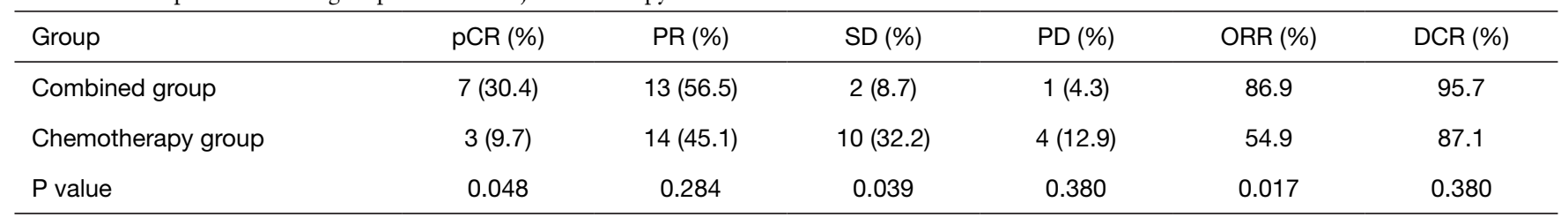

The classified variables were expressed by cases (\%), and the chi-square test or Fisher exact probability method was used. pCR, pathological complete response; PR, partial response; SD, stable disease; PD, progressive disease; ORR, objective response rate; DCR, disease control rate.

Table 3 Comparison of R0 resection rate of esophageal cancer between the two groups

\begin{tabular}{lcc}
\hline Characteristics & $\mathrm{R} 0$ & $\mathrm{R} 1$ \\
\hline Combined group & $21(100.0)$ & $0(0.0)$ \\
Chemotherapy group & $26(96.3)$ & $1(3.7)$ \\
P value & 0.685 & 1.000 \\
\hline
\end{tabular}

The classified variables were expressed by cases (\%), and the chi-square test or Fisher exact probability method was used.

enteritis, immune pneumonia, and other related adverse reactions can be observed in the combination group, but the incidence of above grade 3 was 0 . What is more, there was 1 case of tracheoesophageal fistula in the combined group, 1 case of massive hemorrhage in the simple chemotherapy group, and no death in the two groups (Table 5).

\section{Discussion}

For advanced esophageal cancer, including KEYNOTE-181, ATTRACTION-3, FRONTiER, SHR-1210, etc., studies have confirmed the potential of immunotherapy in the treatment of esophageal cancer. Pembrolizumab, nivolumab, camrelizumab, sintilimab have all been proven to be effective and safe for treatment advanced esophageal, and pembrolizumab is the earliest drug used in immunotherapy and was applied in most clinical trials. In 2019, the ATTRACTION-3 study confirmed the status of secondline immunotherapy combined with chemotherapy for advanced esophageal cancer $(14,15)$. KEYNOTE-590 study showed the efficacy of first-line immunotherapy combined with chemotherapy for advanced ESCC. KEYNOTE-590 study showed that the efficacy of first-line immunotherapy combined with chemotherapy for advanced ESCC was significantly better than that of chemotherapy alone (OS 12.4 months: 9.8 months, $\mathrm{P}<0.0001, \mathrm{HR}=0.73$ ) in
2020. Among the patients with CPS $\geq 10$, the combined immunotherapy group gained the most obvious benefit advantage (13.9 months: 8.8 months, $\mathrm{P}<0.0001$, HR $=0.57)$ (16), which further established the treatment advantage of immunochemotherapy for ESCC. And several clinical trials showed that immunotherapy or combined immunochemotherapy did not significantly increase the incidence of grade 3 or above adverse events (17-20). The latest CheckMate-577 study also presented the obvious benefits of adjuvant immunotherapy after esophageal cancer surgery (21). Compared with placebo adjuvant therapy, nivolumab significantly prolonged the DFS time of patients (22.4 months: 11 months, HR =0.69, $\mathrm{P}=0.0003)$. Therefore, immunotherapy plays an increasingly important role in esophageal cancer.

A study in Japan showed that the expression of PD$\mathrm{L} 1$ and the infiltration of $\mathrm{CD} 8^{+} \mathrm{T}$ cells in tumor tissue were significantly increased in neoadjuvant chemotherapy of locally advanced ESCC (22), suggesting that immunochemotherapy may be more effective in ESCC. It is also based on the great advantages of previous immunochemotherapy that China has carried out several studies on neoadjuvant immunochemotherapy for esophageal cancer (23-26). According to the NICE research results of Professor Yan (24), 11 patients with locally advanced thoracic ESCC were treated with neoadjuvant chemotherapy camrelizumab (albumin paclitaxel + carboplatin). The $\mathrm{R} 0$ resection rate was $100 \%$, and the pathological complete response (pCR) rate was $45.5 \%$ $(5 / 11)$, which was not associated with the expression of PD-L1. In Li's study, the pCR rate was significantly better than in this study. The $\mathrm{N}$ stage was earlier, the treatment response was higher, and the clinical efficacy was better. However, the hematological toxicity of carboplatin was higher than that of nedaplatin, and the toxicity above grade 3 increased significantly. In Gu's KEEP-G 03 study (25), neoadjuvant therapy of sintilimab combined with three 
Table 4 Comparison of tumor regression score between the two groups

\begin{tabular}{lcccccc}
\hline Tumor regression score & 0 & 1 & 2 & 3 & 4 & $\geq 2$ \\
\hline Combined group & $3(14.3)$ & $2(9.5)$ & $5(23.8)$ & $5(23.8)$ & $6(28.6)$ & $16(85.7)$ \\
Chemotherapy group & $6(23.1)$ & $7(26.9)$ & $5(19.2)$ & $5(19.2)$ & $3(11.5)$ & $13(50.0)$ \\
P value & 0.717 & 0.273 & 0.728 & 0.728 & 0.148 & 0.013 \\
\hline
\end{tabular}

The classified variables were expressed by cases (\%), and the chi-square test or Fisher exact probability method was used.

Table 5 Adverse events comparison of the two groups

\begin{tabular}{|c|c|c|c|c|}
\hline Side reactions & \multicolumn{4}{|c|}{ Combination group [chemotherapy group] } \\
\hline Leukopenia & $10[8]$ & $6[7]$ & $3[3]$ & $0[1]$ \\
\hline Neutropenia & 9 [7] & $6[6]$ & $3[3]$ & $0[1]$ \\
\hline Neutropenia with fever & $0[0]$ & $0[0]$ & $1[0]$ & $0[1]$ \\
\hline Thrombocytopenia & $8[6]$ & $5[6]$ & $0[0]$ & $0[0]$ \\
\hline Nausea & 7 [6] & $4[5]$ & $1[1]$ & $0[0]$ \\
\hline Vomiting & $5[5]$ & $3[4]$ & $1[1]$ & $0[0]$ \\
\hline Fatigue & 12 [13] & $7[8]$ & $2[1]$ & $0[0]$ \\
\hline Hypothyroidism or hyperthyroidism & $3[0]$ & $1[0]$ & $0[0]$ & $0[0]$ \\
\hline Rash & $3[0]$ & $2[0]$ & $0[0]$ & $0[0]$ \\
\hline Diarrhea & $2[0]$ & $1[0]$ & $0[0]$ & $0[0]$ \\
\hline Interstitial pneumonia & $0[0]$ & $1[0]$ & $0[0]$ & $0[0]$ \\
\hline Tracheoesophageal fistula & $0[0]$ & $0[0]$ & $0[0]$ & $1[0]$ \\
\hline Hemorrhage & $0[0]$ & $0[0]$ & $0[0]$ & $0[1]$ \\
\hline
\end{tabular}

The chi-square test or Fisher exact probability method was used.

drugs chemotherapies (liposome paclitaxel/cisplatin/tegafur) can help resect ESCC, with an R0 resection rate of $100 \%$, PCR rate of $26.7 \%$, and major pathological response rate of $53.3 \%$, which is better than this trial. This may also be related to the earlier stage of ESCC in Gu's study.

In Nagai's study (27), 141 patients underwent neoadjuvant chemotherapy and subsequent esophagectomy for ESCC were included. A proportion of 7.1 had pathological complete response. Upon endoscopic response evaluation, endoscopic no response, endoscopic partial response, and endoscopic good response were observed in $46(32.6 \%), 54$ (33.3\%), and $41(29.1 \%)$ patients, respectively. Pathological responders significantly increased as the endoscopic response grade became better. Among preoperative clinical factors, only endoscopic response significantly correlated with pathological response in univariate and multivariate analysis. Similar pathological response was found in our chemotherapy group and we will carry on our work on endoscopic response in future. Trials have tried radiotherapy before surgery. It was hoped that this would make the tumour smaller, less likely to spread and patients live longer. However, the review of trials found no clear evidence that patients $\geq 70$ years with locally-advanced esophageal cancer underwent radiotherapy before surgery increases chances of 
survival (28). So in this research we did not enrolled patients underwent radiotherapy.

In this clinical trial, the combined group significantly improved the clinical benefits of ORR, DcR, and pCR; the value of $\mathrm{P}$ showed significantly different. The incidence of adverse events in the two groups was similar, and no serious immune response was found. It is worth promoting the new adjuvant therapy of immunotherapy in the clinical treatment of esophageal cancer. Due to the limited time, PFS and OS cannot be counted. In principle, PFS or OS of the combination group will be longer than the chemotherapy group if the treatment is continued according to guidelines, although it needs further follow-up.

Oncologist hope to find predictors of efficacy of immunotherapy, which can help the options of subsequent treatment several potential biomarkers are being actively investigated, including mismatch-repair deficiency, tumor PD-L1 expression and tumor mutation burden, some of which have been partially validated in clinical trials (29-32). Because of the limited specimen, our study failed to test all these potential biomarkers. In this study, there is a positive correlation on the patient's ORR and the pathological remission rate $(\mathrm{P}=0.017, \mathrm{P}=0.013)$, but no apparent correlation on the changes of DCR and R0 resection rate $(\mathrm{p}=0.380, \mathrm{P}=0.685)$. It indicate that immunotherapy maintenance maybe an optimal choice after surgery, as ATTRACTION-1 (33) demonstrated.

Clinical scientists explore target therapy for ESCC ever, but no molecular-targeting agents demonstrate the clinical utility in Phase 3 trials (34-37) so far. Negative results from Phase 3 trials testing gefitinib and panitumumab suggest the importance of identifying predictive biomarkers of response to molecular-targeting agents $(38,39)$. But next generation sequencing technologies provide comprehensive catalogues of genetic alterations in ESCC may still lead to therapeutic breakthroughs in a personalized manner. And as new combination treatment options emerge, especially combinations of immunotherapy with other agents are expected to lead to enhance immunotherapy efficacy, such as immunochemotherapy, immunoradiotherapy combined with surgery may be breakthroughs treatment in future.

This study is a single-center, and the sample size is small. The expression of PD-L1 is unknown in most patients enrolled in immunotherapy combined with chemotherapy, which cannot be further grouped and analyzed. And because many patients have a short time after the operation, the related adverse reactions after immunotherapy have not appeared, and the complications have not been counted. In conclusion, multicenter, prospective randomized controlled trials are needed to further confirm the efficacy and safety of immunochemotherapy as a neoadjuvant treatment approach for patients with ESCC.

\section{Acknowledgments}

Funding: This study was supported by (I) National Natural Science Foundation of China (NSFC) grant (82172881), (II) Guangdong Basic and Applied Basic Research Foundation (2019A1515011986), (III) Grant of Guangdong Medical Science and Technology Research (A2019420), (IV) the Science and Technology Project Grant of Zhuhai (ZH22036201210067PWC). This work was supported by Investigator Initiated Trials from GCP office of The Fifth Affiliated Hospital of Sun Yat-sen University as following, (I) ChiCTR2000035079. (II) ChiCTR1900022735. PI: ZB. L.

\section{Footnote}

Reporting Checklist: The authors have completed the STROBE reporting checklist. Available at https://dx.doi. org/10.21037/jgo-21-610

Data Sharing Statement: Available at https://dx.doi. org/10.21037/jgo-21-610

Conflicts of Interest: All authors have completed the ICMJE uniform disclosure form (available at https://dx.doi. org/10.21037/jgo-21-610). The authors have no conflicts of interest to declare.

Ethical Statement: The authors are accountable for all aspects of the work in ensuring that questions related to the accuracy or integrity of any part of the work are appropriately investigated and resolved. All procedures performed in this study involving human participants were in accordance with the Declaration of Helsinki (as revised in 2013). The study was approved by ethics board of Fifth Affiliated Hospital of Sun Yat-sen University (No. K248-1) and informed consent was taken from all the patients.

Open Access Statement: This is an Open Access article distributed in accordance with the Creative Commons Attribution-NonCommercial-NoDerivs 4.0 International License (CC BY-NC-ND 4.0), which permits the noncommercial replication and distribution of the article with the strict proviso that no changes or edits are made and the 
original work is properly cited (including links to both the formal publication through the relevant DOI and the license). See: https://creativecommons.org/licenses/by-nc-nd/4.0/.

\section{References}

1. Sung H, Ferlay J, Siegel RL, et al. Global Cancer Statistics 2020: GLOBOCAN Estimates of Incidence and Mortality Worldwide for 36 Cancers in 185 Countries. CA Cancer J Clin 2021;71:209-49.

2. Huang FL, Yu SJ. Esophageal cancer: Risk factors, genetic association, and treatment. Asian J Surg 2018;41:210-5.

3. Ando $\mathrm{N}$, Kato $\mathrm{H}$, Igaki $\mathrm{H}$, et al. A randomized trial comparing postoperative adjuvant chemotherapy with cisplatin and 5 -fluorouracil versus preoperative chemotherapy for localized advanced squamous cell carcinoma of the thoracic esophagus (JCOG9907). Ann Surg Oncol 2012;19:68-74.

4. Tanaka Y, Yoshida K, Tanahashi T, et al. Phase II trial of neoadjuvant chemotherapy with docetaxel, nedaplatin, and $\mathrm{S} 1$ for advanced esophageal squamous cell carcinoma. Cancer Sci 2016;107:764-72.

5. Burmeister BH, Thomas JM, Burmeister EA, et al. Is concurrent radiation therapy required in patients receiving preoperative chemotherapy for adenocarcinoma of the oesophagus? A randomised phase II trial. Eur J Cancer 2011;47:354-60.

6. Cunningham D, Allum WH, Stenning SP, et al. Perioperative chemotherapy versus surgery alone for resectable gastroesophageal cancer. $\mathrm{N}$ Engl J Med 2006;355:11-20.

7. Yamashita K, Mine S, Toihata T, et al. The usefulness of three-dimensional video-assisted thoracoscopic esophagectomy in esophageal cancer patients. Esophagus 2019;16:272-7.

8. Liu S, Wen J, Yang H, et al. Recurrence patterns after neoadjuvant chemoradiotherapy compared with surgery alone in oesophageal squamous cell carcinoma: results from the multicenter phase III trial NEOCRTEC5010. Eur J Cancer 2020;138:113-21.

9. Leng XF, Daiko H, Han YT, et al. Optimal preoperative neoadjuvant therapy for resectable locally advanced esophageal squamous cell carcinoma. Ann N Y Acad Sci 2020;1482:213-24.

10. Yuan X, Liu Y, Li G, et al. Blockade of ImmuneCheckpoint B7-H4 and Lysine Demethylase 5B in Esophageal Squamous Cell Carcinoma Confers Protective Immunity against $\mathrm{P}$. gingivalis Infection. Cancer Immunol
Res 2019;7:1440-56.

11. Li C, Zhao S, Zheng Y, et al. Preoperative pembrolizumab combined with chemoradiotherapy for oesophageal squamous cell carcinoma (PALACE-1). Eur J Cancer 2021;144:232-41.

12. Kojima T, Shah MA, Muro K, et al. Randomized Phase III KEYNOTE-181 Study of Pembrolizumab Versus Chemotherapy in Advanced Esophageal Cancer. J Clin Oncol 2020;38:4138-48.

13. Eisenhauer EA, Therasse P, Bogaerts J, et al. New response evaluation criteria in solid tumours: revised RECIST guideline (version 1.1). Eur J Cancer 2009;45:228-47.

14. Chirieac LR, Swisher SG, Ajani JA, et al. Posttherapy pathologic stage predicts survival in patients with esophageal carcinoma receiving preoperative chemoradiation. Cancer 2005;103:1347-55.

15. Yamamoto S, Kato K, Daiko H, et al. Feasibility study of nivolumab as neoadjuvant chemotherapy for locally esophageal carcinoma: FRONTiER (JCOG1804E). Future Oncol 2020;16:1351-7.

16. Kato K, Cho BC, Takahashi M, et al. Nivolumab versus chemotherapy in patients with advanced oesophageal squamous cell carcinoma refractory or intolerant to previous chemotherapy (ATTRACTION-3): a multicentre, randomised, open-label, phase 3 trial. Lancet Oncol 2019;20:1506-17.

17. Kato K, Shah MA, Enzinger P, et al. KEYNOTE-590: Phase III study of first-line chemotherapy with or without pembrolizumab for advanced esophageal cancer. Future Oncol 2019;15:1057-66.

18. Gadgeel S, Rodríguez-Abreu D, Speranza G, et al. Updated Analysis From KEYNOTE-189: Pembrolizumab or Placebo Plus Pemetrexed and Platinum for Previously Untreated Metastatic Nonsquamous Non-Small-Cell Lung Cancer. J Clin Oncol 2020;38:1505-17.

19. Shah MA, Kojima T, Hochhauser D, et al. Efficacy and Safety of Pembrolizumab for Heavily Pretreated Patients With Advanced, Metastatic Adenocarcinoma or Squamous Cell Carcinoma of the Esophagus: The Phase 2 KEYNOTE-180 Study. JAMA Oncol 2019;5:546-50.

20. Kudo T, Hamamoto Y, Kato K, et al. Nivolumab treatment for oesophageal squamous-cell carcinoma: an open-label, multicentre, phase 2 trial. Lancet Oncol 2017;18:631-9.

21. Seiwert TY, Burtness B, Mehra R, et al. Safety and clinical activity of pembrolizumab for treatment of recurrent or metastatic squamous cell carcinoma of the head and neck (KEYNOTE-012): an open-label, multicentre, phase 1b trial. Lancet Oncol 2016;17:956-65. 
22. Kelly RJ, Ajani JA, Kuzdzal J, et al. Adjuvant Nivolumab in Resected Esophageal or Gastroesophageal Junction Cancer. N Engl J Med 2021;384:1191-203.

23. Fukuoka E, Yamashita K, Tanaka T, et al. Neoadjuvant Chemotherapy Increases PD-L1 Expression and CD8+ Tumor-infiltrating Lymphocytes in Esophageal Squamous Cell Carcinoma. Anticancer Res 2019;39:4539-48.

24. Yan MH, Hou XB, Cai BN, et al. Neoadjuvant chemoradiotherapy plus surgery in the treatment of potentially resectable thoracic esophageal squamous cell carcinoma. World J Clin Cases 2020;8:6315-21.

25. Gu Y, Chen X, Wang D, et al. A study of neoadjuvant sintilimab combined with triplet chemotherapy of lipopaclitaxel, cisplatin, and S-1 for resectable esophageal squamous carcinoma (ESCC). Ann Oncol 2020;31:1307-8.

26. Zhang G, Hu Y, Yang B, et al. A single-centre, prospective, open-label, single-arm trial of toripalimab with nabpaclitaxel and S-1 as a neoadjuvant therapy for esophageal squamous cell carcinoma (ESCC). Ann Oncol 2020;31:722.

27. Nagai Y, Yoshida N, Baba Y, et al. Clinical significance of evaluating endoscopic response to neoadjuvant chemotherapy in esophageal squamous cell carcinoma. Dig Endosc 2020;32:39-48.

28. Arnott SJ, Duncan W, Gignoux M, et al. Preoperative radiotherapy for esophageal carcinoma. Cochrane Database Syst Rev 2005 ;(4):CD001799.

29. Reck M, Rodríguez-Abreu D, Robinson AG, et al. Pembrolizumab versus Chemotherapy for PD-L1Positive Non-Small-Cell Lung Cancer. N Engl J Med 2016;375:1823-33.

30. Hellmann MD, Ciuleanu TE, Pluzanski A, et al. Nivolumab plus Ipilimumab in Lung Cancer with a High Tumor Mutational Burden. N Engl J Med 2018;378:2093-104.

31. Hellmann MD, Callahan MK, Awad MM, et al. Tumor Mutational Burden and Efficacy of Nivolumab

Cite this article as: Huang $\mathrm{B}$, Shi $\mathrm{H}$, Gong X, Yu J, Xiao C, Zhou B, Liang Z, Li X. Comparison of efficacy and safety between pembrolizumab combined with chemotherapy and simple chemotherapy in neoadjuvant therapy for esophageal squamous cell carcinoma. J Gastrointest Oncol 2021;12(5):20132021. doi: 10.21037/jgo-21-610
Monotherapy and in Combination with Ipilimumab in Small-Cell Lung Cancer. Cancer Cell 2018;33:853-861.e4.

32. Le DT, Uram JN, Wang H, et al. PD-1 Blockade in Tumors with Mismatch-Repair Deficiency. N Engl J Med 2015;372:2509-20.

33. Satoh T, Kato K, Ura T, et al. Five-year follow-up of nivolumab treatment in Japanese patients with esophageal squamous-cell carcinoma (ATTRACTION-1/ONO-453807). Esophagus 2021;18:835-43.

34. Dutton SJ, Ferry DR, Blazeby JM, et al. Gefitinib for oesophageal cancer progressing after chemotherapy (COG): a phase 3, multicentre, double-blind, placebo-controlled randomised trial. Lancet Oncol 2014;15:894-904.

35. Petty RD, Dahle-Smith A, Stevenson DAJ, et al. Gefitinib and EGFR Gene Copy Number Aberrations in Esophageal Cancer. J Clin Oncol 2017;35:2279-87.

36. Ilson DH, Kelsen D, Shah M, et al. A phase 2 trial of erlotinib in patients with previously treated squamous cell and adenocarcinoma of the esophagus. Cancer 2011;117:1409-14.

37. Lorenzen S, Schuster T, Porschen R, et al. Cetuximab plus cisplatin-5-fluorouracil versus cisplatin-5-fluorouracil alone in first-line metastatic squamous cell carcinoma of the esophagus: a randomized phase II study of the Arbeitsgemeinschaft Internistische Onkologie. Ann Oncol 2009;20:1667-73.

38. Li S, Wang Z, Huang J, et al. Clinicopathological and prognostic significance of mTOR and phosphorylated mTOR expression in patients with esophageal squamous cell carcinoma: a systematic review and meta-analysis. BMC Cancer 2016;16:877.

39. Kato K,Doi T,Kojima T,et al.Phase II study of BKM 120 in patients with advanced esophageal squamous cell carcinoma (EPOC1303).Am Soc Clin Onco 12017.

(English Language Editor: J. Chapnick) 